\title{
MS elps.201300100.R1
}

20 March 2013

$\sim 3500$ words

\section{Electrophoresis}

\section{Genetic characterization of selected parasites from people with histories of gastrointestinal disorders using a mutation scanning- coupled approach}

\author{
Anson V. Koehler ${ }^{1 *}$ \\ Richard S. Bradbury ${ }^{2,3}$ \\ Melita A. Stevens ${ }^{4}$ \\ Shane R. Haydon ${ }^{4}$ \\ Aaron R. Jex ${ }^{1}$ \\ Robin B. Gasser ${ }^{1 * *}$
}

${ }_{1}^{1}$ Faculty of Veterinary Science, The University of Melbourne, Victoria, Australia
2 Medical Research Council International Nutrition Group, Keneba, The Gambia
${ }^{3}$ School of Medicine, University of Tasmania, Hobart, Tasmania, Australia
${ }^{4}$ Melbourne Water Corporation, Victoria, Australia

*Dr Anson Koehler, The University of Melbourne, Faculty of Veterinary Sciences, Corner Park Drive and Flemington Road, Parkville, Victoria 3010, Australia. Email: anson.koehler@unimelb.edu.au. Tel.: +61 39731 2295. Fax: +61 383447374

**Additional corresponding author:
Professor Robin B. Gasser; E-mail: robinbg@ unimelb.edu.au

Abbreviations: BI, Bayesian inference; gp60, 60-kDa glycoprotein; ITS-2, second internal transcribed spacer of nuclear ribosomal DNA; L3s, third-stage larvae; Nst, number of substitutions; pgp60, part of the 60-kDa glycoprotein gene; pp, posterior probability; ptpi, part of the triose phosphate isomerase gene; REF, restriction endonuclease fingerprinting; STH, soil-transmitted helminth; tpi, triose phosphate isomerase gene; WHO, World Health Organization.

Received: February 25, 2013; Revised: March 21, 2013; Accepted: March 22, 2013

This article has been accepted for publication and undergone full peer review but has not been through the copyediting, typesetting, pagination and proofreading process, which may lead to differences between this version and the Version of Record. Please cite this article as doi: 10.1002/elps.201300100 


\section{[Abstract]}

A single-strand conformation polymorphism (SSCP) analysis and targeted sequencing approach was used for the genetic characterization of some major pathogens from a cohort of 227 people with histories of gastrointestinal disorders. Genomic DNAs from faecal samples were subjected to PCR-amplification of regions in the glycoprotein (gp60) or triose phosphate isomerase (tpi) gene, or the second internal transcribed spacer of nuclear ribosomal DNA (ITS-2). Cryptosporidium, Giardia and strongylid nematodes were detected in 94, 132 and 12 samples. Cryptosporidium hominis subgenotypes IbA10G2, IdA15G1, IgA17, IgA18 and IfA13G1 were identified in $74.6,16.9,5.6,1.4$ and $1.4 \%$ of 71 samples, respectively. For C. parvum, subgenotypes IIaA17G2R1 (47.6\%) and IIaA18G3R1 (23.8\%) were identified in 23 samples. Giardia duodenalis assemblage B (78\%) was more common than assemblage A (22\%). In addition, DNA of the nematodes Ancylostoma ceylanicum ( $\mathrm{n}=2)$, Ancylostoma duodenale (4), Necator americanus (5) and Haemonchus contortus (1) was specifically detected. This is the first report of A. ceylanicum in two persons in Australia and, we provide molecular evidence of $H$. contortus in a child. This SSCP-based approach should provide a useful diagnostic and analytical tool for a wide range of pathogens.

\section{Keywords:}

Cryptosporidium / Giardia / hookworm / pathogen / single-strand conformation polymorphism (SSCP) analysis.

\section{Introduction}

Gastrointestinal parasites of humans cause destructive diseases of major socio-economic importance worldwide. For instance, in the developed world, waterborne protists, such as Cryptosporidium and Giardia, can be responsible for up to 20-30\% of cases of diarrhoea in some countries [1]. Diarrhoea has been recognized by the World Health Organization (WHO) as the second leading cause of death in children, and the leading cause of malnutrition in children of less than five years of age [2]. In 2004, Giardia and Cryptosporidium were added to the WHO's 'Neglected Diseases Initiative' [3] in an effort to boost the global profile and improve the detection and control of these pathogens. Parasitic worms (i.e., trematodes, cestodes and nematodes) have a massive, long-term impact on human health and cause substantial suffering (e.g., [4-9]). WHO estimates that 2.9 billion people are infected with nematodes [10] and that morbidity from worms surpasses diabetes and lung cancer in disability adjusted life years (DALYs) [11]. Soil-transmitted helminths (STHs), such as the hookworms Necator americanus and Ancylostoma duodenale, are of major importance, infecting 740 million people and causing anaemia and malnutrition worldwide [12].

The diagnosis of parasitic infections and diseases is central to investigating their epidemiology, treatment and control. Methods routinely used for parasite diagnosis mostly 
rely on the microscopic detection of parasite stages (e.g., [1, 13-16]). These approaches can be time-consuming to perform and are quite often unreliable [17, 18]. For instance, using coproscopic methods, it is usually not possible to identify or distinguish different parasite species based on the morphology of the structures detected (e.g., oocysts, cysts or eggs), because of an absence or lack of distinguishing morphological features. In addition, immunological assays, such as commercial coproantigen detection methods, are often not entirely specific (e.g., [19-24]). The limited specificity and/or sensitivity of some of these phenetic methods represent a major constraint for the routine diagnosis of parasitic infections.

The use of DNA-based methods as complementary tools can circumvent this limitation. Particularly polymerase chain reaction (PCR)-coupled methods are useful for the genetic identification and characterization of pathogens from tiny amounts (pg to fg) of parasite DNA $[25,26]$. Although a wide range of PCR-coupled methods is available, mutation scanning [27] provides a powerful tool for both diagnosis and genetic analysis, provided suitable molecular markers are employed. In particular, single-strand conformation polymorphism (SSCP) has found applicability because a standard protocol can be applied to a wide range of genetic markers and organisms. For instance, PCR-based SSCP analysis of internal transcribed spacer sequences of nuclear ribosomal DNA allows the specific identification of key parasitic nematodes of major importance [27] and nuclear genes, such as $60-\mathrm{kDa}$ glycoprotein ( $\mathrm{gp} 60)$ and triose phosphate isomerase (tpi), are commonly employed for the specific detection or characterization of protozoa, such as Cryptosporidium [21, 28-34] and Giardia [35, 36]. The method has been applied to DNA isolated directly from parasitic protozoans and from biological matrices, including faeces.

Although SSCP has been used for systematic, epidemiological and population genetic investigations of pathogens [27, 37], its dual diagnostic/analytical capacity has not been fully exploited in a pathology, microbiology or parasitology laboratory setting to complement routine diagnostic testing (using phenetic, including immunological, methods). In the present study, we employed PCR-coupled SSCP analysis and targeted sequencing to genetically characterize key pathogens in the faeces from patients with histories of gastrointestinal disorders. The main focus here was on the protozoa Cryptosporidium and Giardia as well as strongylid nematodes, such as species of Ancylostoma and Necator (hookworms), because of their major socioeconomic impact.

\section{Materials and methods}

\subsection{Samples and conventional diagnostic testing}

A total of 227 faecal samples from anonymous humans with histories of gastrointestinal disorders were tested in two diagnostic pathology service laboratories in Australia (between Mar. 2010 and Nov. 2011), and aliquots thereof provided to us for genetic testing. Most samples $(\mathrm{n}=217)$ were from localities in Western Australia, five were from the Northern Territory, and five were from unknown localities in Australia. The faecal samples had been tested for the presence of parasitic protozoa and helminths using conventional methods. Cysts and oocysts of Giardia and Cryptosporidium, respectively, were detected microscopically either by a formalin-ethyl acetate concentration technique [38], immunofluorescence [39], modified acid-fast staining [40] or using a commercial coproantigen immunoassay (CELISA, Cellabs, Australia). Eggs of strongylid nematodes (i.e. of the order Strongylida) were detected microscopically following the formalin-ethyl acetate concentration method [15]. Following conventional testing, the remaining amounts of individual faecal samples were stored at $-80{ }^{\circ} \mathrm{C}$. 


\subsection{Testing of DNA isolated from faecal samples by PCR-coupled SSCP analysis and targeted sequencing}

\subsubsection{Genomic DNA isolation and PCR}

Genomic DNA was extracted directly from 0.1 to $0.4 \mathrm{~g}$ of individual faecal samples using the PowerSoil DNA Isolation Kit (MoBio, USA), according to the manufacturer's protocol. This kit was used because it is known to be effective at removing components that are inhibitory to PCR [41, 42]. Following DNA isolation, individual genomic DNA samples were subjected to nested or single-step PCR, targeting different regions of nuclear DNA. Each PCR was carried out in a $50 \mu \mathrm{l}$ volume containing $10 \mathrm{mM}$ Tris- $\mathrm{HCl}(\mathrm{pH} 8.4), 50 \mathrm{mM} \mathrm{KCl}$ (Promega, USA), $3.0 \mathrm{mM}$ of $\mathrm{MgCl}_{2}, 200 \mu \mathrm{M}$ of each deoxynucleotide triphosphate, $100 \mathrm{pmol}$ of each primer and $1 \mathrm{U}$ of GoTaq (Promega) DNA polymerase.

For human-infective Cryptosporidium, the gp60 gene $(\sim 1 \mathrm{~kb})$ was amplified using primers gp15-ATG (forward: 5'-ATGAGATTGTCGCCTCATTATC-3') and gp15-STOP (reverse: 5'-TTACAACACGAATAAGGCTGC-3') [43], followed by the nested amplification of a portion of the gp60 gene (designated pgp60; 250-350 bp) using primers gp15-15A (forward: 5'-GCCGTTCCACTCAGAGGAAC-3') and gp15-15E (reverse: 5'CCACATTACAAATGAAGTGCCGC-3') [44]. Primary amplification of gp60 utilized the cycling protocol: $94{ }^{\circ} \mathrm{C}$ for 5 min (initial denaturation), followed by 35 cycles of $94{ }^{\circ} \mathrm{C}$ for 30 $\sec$ (denaturation), $55^{\circ} \mathrm{C}$ for $45 \mathrm{sec}$ (annealing) and $72{ }^{\circ} \mathrm{C}$ for $1 \mathrm{~min}$ (extension), with a final extension of $72{ }^{\circ} \mathrm{C} / 10 \mathrm{~min}$. For the secondary amplification of pgp60, we employed $94{ }^{\circ} \mathrm{C}$ for $5 \mathrm{~min}$, followed by $30 \mathrm{cycles}$ of $94{ }^{\circ} \mathrm{C}$ for $30 \mathrm{sec}, 55^{\circ} \mathrm{C}$ for $30 \mathrm{sec}$ and $72{ }^{\circ} \mathrm{C}$ for $30 \mathrm{sec}$, with a final extension at $72{ }^{\circ} \mathrm{C}$ for $10 \mathrm{~min}$.

For Giardia, the tpi gene of Giardia was amplified using primers AL3543 (forward: 5'AAATTATGCCTGCTCGTCG-3') and AL3546 (reverse: 5'CAAACCTTTTCCGCAAACC-3'), followed by nested amplification of a portion of the tpi gene (called ptpi; $\sim 530$ bp) employing primers AL3544 (forward: 5'CCCTTCATCGGTGGTAACTT-3') and AL3545 (reverse: 5'GTGGCCACCACTCCCGTGCC-3') [45]. For the primary amplification, the cycling protocol was $94{ }^{\circ} \mathrm{C}$ for $5 \mathrm{~min}$ (initial denaturation), followed by 35 cycles of $94{ }^{\circ} \mathrm{C}$ for $45 \mathrm{sec}$ (denaturation), $50{ }^{\circ} \mathrm{C}$ for $45 \mathrm{sec}$ (annealing) and $72{ }^{\circ} \mathrm{C}$ for $1 \mathrm{~min}$ (extension), with a final extension of $72{ }^{\circ} \mathrm{C}$ for $10 \mathrm{~min}$. Secondary amplification of ptpi was achieved employing 94 ${ }^{\circ} \mathrm{C}$ for $5 \mathrm{~min}$, followed by 35 cycles of $94{ }^{\circ} \mathrm{C}$ for $45 \mathrm{sec}, 55^{\circ} \mathrm{C}$ for $30 \mathrm{sec}$, and $72{ }^{\circ} \mathrm{C}$ for 1 min, with a final extension at $72^{\circ} \mathrm{C}$ for $10 \mathrm{~min}$.

For strongylid nematodes (including human hookworms), the second internal transcribed spacer (ITS-2; 310-420 bp) was amplified using primers NC1 (forward: 5'ACGTCTGGTTCAGGGTTGTT-3') and NC2 (reverse: 5'-TTAGTTTCTTTTCCTCCGCT$\left.3^{\prime}\right)[27,46]$. The cycling protocol was: $94^{\circ} \mathrm{C}$ for $5 \mathrm{~min}$ (initial denaturation), followed by 35 cycles of $94{ }^{\circ} \mathrm{C}$ for $30 \mathrm{sec}$ (denaturation), $55^{\circ} \mathrm{C}$ for $30 \mathrm{sec}$ (annealing) and $72{ }^{\circ} \mathrm{C}$ for $30 \mathrm{sec}$ (extension), with a final extension of $72{ }^{\circ} \mathrm{C}$ for $5 \mathrm{~min}$.

\subsubsection{Agarose gel electrophoretic analysis of amplicons}

The quality, intensity and size of individual amplicons were examined on ethidium bromidestained $1.5 \%$ agarose gels, using TBE (65 mM Tris-HCl, $27 \mathrm{mM}$ boric acid, $1 \mathrm{mM}$ EDTA, pH 9; Bio-Rad, USA) as the buffer and ФX174-HaeIII (Promega, USA) as a size marker. Amplicons were then subjected to non-isotopic SSCP or restriction endonuclease fingerprinting (REF) analysis (see section 2.2.3). 


\subsubsection{SSCP and targeted sequencing}

Direct SSCP (protocol B; [27]) was used for the analysis of pgp60 (Cryptosporidium) and ITS-2 (strongylid nematodes) amplicons. In brief, $1 \mu \mathrm{l}$ of each secondary amplicon was mixed with $5 \mu \mathrm{l}$ sequencing-stop solution (Promega) and, heat-denatured at $94{ }^{\circ} \mathrm{C} / 30 \mathrm{~min}$, snap-cooled on a freeze-block $\left(-20^{\circ} \mathrm{C}\right)$ and then subjected to electrophoresis at $74 \mathrm{~V}$ at 7.4 ${ }^{\circ} \mathrm{C}$ (constant) for 16-18 h in a GMA Wide Mini S-2x25 gel in a SEA 2000 rig (Elchrom Scientific AG) using TAE buffer (40 mM Tris base, $20 \mathrm{mM}$ acetic acid, $1.0 \mathrm{mM}$ EDTA, Bio$\mathrm{Rad}$ ). Control samples (representing known species or genotypes) were included on each gel to ensure the reproducibility of profiles representing this sample among gels.

REF [35, 47] was used for the analysis of ptpi amplicons, because their size ( 530 bp) exceeded that usually employed for direct SSCP analysis. In brief, an aliquot (10 $\mu 1)$ of each amplicon was cleaved with $10 \mathrm{IU}$ of the restriction endonuclease HaeIII (Promega, USA) in $20 \mu \mathrm{l}$, incubated at $37^{\circ} \mathrm{C}$ for $2 \mathrm{~h}$, and the enzyme inactivated at $70{ }^{\circ} \mathrm{C}$ for $5 \mathrm{~min}$. One $\mu \mathrm{l}$ of this digest was then subjected to analysis by SSCP (protocol B; [27]) using the same conditions as for amplicons of $\leq 450 \mathrm{bp}$.

Following these SSCP-based analyses, selected ptpi $(\mathrm{n}=52)$, pgp60 $(\mathrm{n}=33)$ and ITS-2 $(\mathrm{n}=5)$ amplicons representing each distinct electrophoretic profile were treated with ExoSAP-IT $^{\circledR}$ (Affymetrix, USA), according to the manufacturer's instructions, and then subjected to direct, automated sequencing (BigDye Terminator v.3.1 chemistry, Applied Biosystems, USA) using the same primers employed in the prior PCR. Sequence quality was verified by comparison with corresponding electro-pherograms using Geneious v.6.0.4 (Biomatters, New Zealand).

\subsubsection{Phylogenetic analysis of sequence data}

Representative sequences were aligned with 12 (C. parvum pgp60), 6 (C. hominis pgp60), 13 (strongylid ITS-2) or 13 (Giardia ptpi) reference sequences (Supplementary Tables S1-S4). Sequences were aligned using the program ClustalW [48], and alignments adjusted manually using Mesquite v.2.75 [49]. Phylogenetic analysis of sequence data was conducted by Bayesian inference (BI) using Monte Carlo Markov Chain analysis in the program MrBayes v.3.1.2 [50]. The likelihood parameters set for the BI analysis of sequence data were based on the Akaike Information Criteria test in jModeltest v.2.1 [51]. For the separate analyses of pgp60 (C. parvum), ptpi (Giardia) and ITS-2 (strongylid nematodes) sequence data, the number of substitutions (Nst) was set at 6, with a gamma distribution. For the pgp60 sequence data of $C$. hominis, Nst was also set at 6, using a proportion of invariable sites. Posterior probability (pp) values were calculated by running 2,000,000 generations with four simultaneous tree-building chains. Trees were saved every $100^{\text {th }}$ generation. At the end of each run, the standard deviation of split frequencies was $<0.01$, and the potential scale reduction factor approached one. A 50\% majority rule consensus tree for each analysis was constructed based on the final $75 \%$ of trees generated by BI. Analyses were run three times to ensure convergence and insensitivity to priors. Outgroups used in the analyses were $C$. hominis for C. parvum (pgp60), C. parvum for C. hominis (pgp60), Giardia ardeae and $G$. muris for G. duodenalis (ptpi) and Oesphagostomum dentatum for strongylid nematodes (ITS-2). 


\section{Results and discussion}

Using three distinct PCR assays, amplicons were produced from 227 genomic DNAs from individual faecal samples from humans with histories of gastrointestinal disorders (including diarrhoea, abdominal pain, vomiting and/or anaemia) and known to be passing Cryptosporidium oocysts, Giardia cysts and/or strongylid nematode eggs, based on conventional copro-diagnostic testing. In total, 88 samples were PCR test-positive for Cryptosporidium, 122 for Giardia, 13 for both Cryptosporidium and Giardia, 11 for one or more strongylid nematodes, and one sample for both Giardia and at least a strongylid nematode. All of the amplicons were subjected to SSCP (see Fig. 1) or REF analysis. All SSCP profiles were recorded, and multiple amplicons representing each individual profile selected for subsequent sequencing.

\subsection{Cryptosporidium}

Amplicons (pgp60) were amplified by PCR from 94 DNA samples. Following SSCP analysis and selective sequencing, $71(75.5 \%), 21(22.3 \%)$ and two $(2.1 \%)$ of the 94 DNA samples represented $C$. hominis, $C$. parvum and $C$. meleagridis, respectively. Phylogenetic analysis of the nucleotide sequence data showed that $C$. hominis was represented by genotypes $\mathrm{Ib}, \mathrm{Id}, \mathrm{Ig}$ and If, with subgenotype IbA10G2 being most prevalent $(\mathrm{n}=53 ; 74.6 \%)$ (Table 1 ; Fig. 2). $C$. parvum was represented by genotype IIa, with IIaA18G3R1 being the commonest subgenotype (Table 1; Fig. 3). C. meleagridis was represented by a previously published reference sequence (GenBank accession number JX548300). There was no significant difference between the number of male and female patients excreting $C$. hominis or $C$. parvum (goodness-of-fit test: $\mathrm{P}=0.561$ and $\mathrm{P}=0.664$, respectively) (Table 2). Cryptosporidium was found predominantly in patients of $\leq 9$ years of age (Table 2).

The numbers of samples PCR-test positive for C. hominis $(75.5 \%)$ and $C$. parvum (22.3\%) were comparable with those reported previously in Australia [21, 52-55] and other countries (reviewed by Xiao et al. [56]), and are consistent with the finding that the majority of human cryptosporidiosis cases in industrialized countries relate to C. hominis [56]. To date, IbA10G2 is the commonest subgenotype recorded in humans worldwide [57]. A previous study of samples from humans from Western Australia showed that IdA15G1 was the main subgenotype [53], whereas, here, C. hominis IbA10G2 dominated. This discrepancy might relate to a significant regional outbreak of subgenotype IdA15G1 in 2010 in Western Australia [53]. For C. parvum, the two most dominant subgenotypes recorded here were IIaA17G2R1 and IIaA18G3R1, which is in accordance with previous findings in Australia [53].

The two patients with $C$. meleagridis were both one-year-old females presenting with diarrhoea, but there was no evidence of other, concurrent parasitic infections. There are numerous published reports of $C$. meleagridis in humans around the world [58]. Mostly, this species of Cryptosporidium is detected in children and immuno-compromised or -suppressed persons who have had direct or indirect contact with infected birds, such as turkeys or chickens [59]. Although $C$. meleagridis has a specific affiliation with birds, it is directly transmissible to humans and can be transmitted among humans, particularly those suffering from immunological disorders [58].

\subsection{Giardia}

Amplicons (ptpi) were produced by PCR from 136 DNA samples. Following REF analysis and selective sequencing, 103 amplicons represented assemblage B (78\%), and 29 belonged 
to assemblage A (22\%). A subset of 25 amplicons representing unique profiles was subjected to sequencing, and all of the (distinct) sequence types subjected to phylogenetic analysis. The tree showed that all samples belonged to either assemblage A or B (Fig. 4), and a pairwise comparison of all sequences revealed that assemblage A was represented by two distinct sequence types, and assemblage B by 23 distinct types. Of these 25 ptpi sequences, eight were present in the GenBank database and, interestingly, the remaining 17 are new sequence types differing by 1-4 bp from previously published sequences. The number of male patients with $G$. duodenalis was significantly greater than that of females (binomial goodness-of-fit test: $\mathrm{P}=0.037$ ) (Table 2). G. duodenalis was detected predominantly in patients of $\leq 9$ years of age (Table 2).

G. duodenalis assemblage B was far more frequently detected than assemblage A (78\% and $22 \%$, respectively). The predominance of assemblage B is concordant with the findings from studies of Giardia/giardiasis of humans in Australia ([60]: assemblage B $=75 \%$; assemblage $\mathrm{A}=25 \% ; \mathrm{n}=124 ;$ [61]: assemblage $\mathrm{B}=70 \%$; assemblage $\mathrm{A}=30 \% ; \mathrm{n}=23$ ). Worldwide, based on current evidence, assemblage B appears to be only slightly more prevalent than assemblage $\mathrm{A}$ in both developed and developing countries [62]. Although a higher (apparent) prevalence of this assemblage might relate to higher reproductive potential and faecal cyst counts compared with assemblage A [63], there is presently no clear evidence to indicate that assemblage A is more pathogenic than assemblage B [62]. This statement is supported by the study of Lebbad et al. [64] who did not find an association between particular assemblages and clinical symptoms in 214 patients with giardiasis in Sweden.

\subsection{Nematodes}

Amplicons (ITS-2) were produced from 12 of the 227 genomic DNA samples. SSCP analysis revealed four distinct profiles. Following sequencing, the hookworms Ancylostoma ceylanicum ( $\mathrm{n}=2$ ), Ancylostoma duodenale (4) and Necator americanus (5) were detected, and the barber's pole worm, Haemonchus contortus (1), which only usually infects small ruminants, such as sheep and goats (Fig. 5).

Both A. duodenale and N. americanus have been reported in Australia, and were first recorded during the Australian Hookworm Campaign (1919-1924) [65]. Originally, the dominant species in Queensland and New South Wales was N. americanus, but in more recent times only $A$. duodenale has been documented in indigenous communities of Western Australia and the Northern Territory $[65,66]$. In the present study, all five people inferred to harbour N. americanus likely imported this parasite into Australia from overseas, because all patients were recent immigrants from Sierra Leone or refugees from Sudan where $N$. americanus is endemic [67, 68].

In addition, the first two cases of A. ceylanicum in humans were recorded in Western Australia. These people were both in their mid twenties, one from Perth the other from the Pilbara region. Ancylostoma ceylanicum is the only hookworm of dogs and cats that can be transmitted to humans and cause patent intestinal infection [69, 70]. Recently, A. ceylanicum has been found in dogs from Western Australia, Queensland and the Northern Territory [71]. Although it possible that these people might have acquired this hookworm outside of Australia, there was no documented evidence of travel; therefore, it is more likely that they became infected in Australia by A. ceylanicum derived from dogs or cats.

Interestingly, $H$. contortus DNA was amplified from a faecal sample from a one-yearold female child, which is the first case of $H$. contortus detected by molecular means in a human. This particular patient presented with diarrhoea, a cough and fever and might have been immuno-compromised, as she was shown to harbour mixed infections of Blastocystis hominis, Chilomastix mesnili, Endolimax nana and Entamoeba coli by coproscopy, although 
no helminth eggs were detected upon the microscopic examination. Globally, there are seven documented cases of $H$. contortus infection in humans, following the detection of either eggs or worms in faecal samples [72, 73]. Most of these cases are believed to relate to humans accidentally ingesting infective third-stage larvae (L3s) from environments stocked with large numbers of small ruminants (including sheep and goats) infected with $H$. contortus [73]. This child may have become infected or been exposed to H. contortus L3s or eggs through coprophagia (ingestion of faecal matter), or geophagia (ingestion of soil), recognized as a significant risk factor for children (between 1-3 years of age) acquiring diseases [74]. The present case might also be the result of pseudo-parasitism, whereby PCR detected $H$. contortus DNA originating from L3s or eggs accidentally ingested and passing through the gastrointestinal tract of the non-permissive human host, but not relating to an actual infection. Interestingly, three of all seven previously published cases of $H$. contortus infection in humans worldwide relate to indigenous persons from Western Australia [73].

\section{Concluding remarks}

PCR-based SSCP analysis and targeted sequencing allowed the genetic characterization of major pathogens from a cohort of 227 people with histories of gastrointestinal disorders. Cryptosporidium and Giardia were characterized to the genotypic/assemblage and subgenotypic levels by comparison with homologous reference sequences from previous national and global studies, accessible from the GenBank database. Although no novel genotypes or assemblages of Cryptosporidium and Giardia were discovered, two interesting cases of $C$. meleagridis (typically found in birds) were identified in faecal samples from children. We detected, for the first time, A. ceylanicum in two persons from Australia that most likely originated from infected dogs or cats. In addition, we provided molecular evidence of $H$. contortus exposure or infection in a young child, probably via a small ruminant source.

Although the SSCP-based analyses have been used routinely in our laboratory for the detection and characterization of species and genotypes/assemblages of Cryptosporidium or Giardia [21, 29, 31-36, 75, 76], it had not been employed previously for the analysis of ITS-2 products PCR-amplified directly from human faecal DNA samples (rather than parasite DNA). The detection of single ITS-2 bands on agarose gels, discrete profiles by SSCP and unambiguous sequences resulting from selective sequencing of amplicons has inferred exquisite specificity of the PCR (determined by primer NC1). Therefore, this SSCP-based approach should find significant utility as an epidemiology tool for the simultaneous diagnosis and genetic analysis of hookworms and other strongylid nematodes of humans.

Nucleotide sequences reported in this paper are available from the GenBank database under accession nos. KC632529-KC632571.

This project was funded by the Australian Research Council (ARC), the National Health and Medical Research Council (NHMRC), and Melbourne Water Corporation. This project was also supported by a Victorian Life Sciences Computation Initiative (VLSCI) grant number VR0007 on its Peak Computing Facility at The University of Melbourne, an initiative of the Victorian Government. Other support from the Alexander von Humboldt Foundation (RBG) and the IBM Research Collaboratory for Life Sciences - Melbourne is gratefully acknowledged. We thank the PathWest Enteric Reference Laboratory and Western Diagnostic Pathology for providing samples.

The authors have no conflict of interest. 


\section{References}

[1] Fletcher, S. M., Stark, D., Harkness, J., Ellis, J., Clin. Microbiol. Rev. 2012, 25, 420449.

[2] Kosek, M., Bern, C., Guerrant, R. L., Bull. World Health Organ. 2003, 81, 197-204.

[3] Savioli, L., Smith, H., Thompson, A., Trends Parasitol. 2006, 22, 203-208.

[4] Eckert, J., Deplazes, P., Clin. Microbiol. Rev. 2004, 17, 107-135.

[5] Hotez, P. J., Brindley, P. J., Bethony, J. M., King, C. H., Pearce, E. J., Jacobson, J., J. Clin. Invest. 2008, 118, 1311-1321.

[6] Pullan, R., Brooker, S., Parasitology 2008, 135, 783-793.

[7] Davidson, R. K., Romig, T., Jenkins, E., Tryland, M., Robertson, L. J., Trends Parasitol. 2012, 28, 239-247.

[8] Fürst, T., Keiser, J., Utzinger, J., Lancet Infect. Dis. 2011, 12, 210-221.

[9] Fürst, T., Sayasone, S., Odermatt, P., Keiser, J., Utzinger, J., BMJ 2012, 344, 1-11.

[10] Hotez, P. J., Molyneux, D. H., Fenwick, A., Kumaresan, J., Sachs, S. E., Sachs, J. D., Savioli, L., N. Engl. J. Med. 2007, 357, 1018-1027.

[11] Hotez, P. J., Bethony, J., Bottazzi, M. E., Brooker, S., Diemert, D., Loukas, A., Trends Parasitol. 2006, 22, 327-331.

[12] Bethony, J., Brooker, S., Albonico, M., Geiger, S. M., Loukas, A., Diemert, D., Hotez, P. J., Lancet 2006, 367, 1521-1532.

[13] Jex, A. R., Pangasa, A., Campbell, B. E., Whipp, M., Hogg, G., Sinclair, M. I., Stevens, M., Gasser, R. B., J. Clin. Microbiol. 2008, 46, 2252-2262.

[14] Chalmers, R., in: Ortega-Pierres, G., Cacciò, S., Fayer, R., Mank, T., Smith, H., Thompson, R. (Eds.), Giardia and Cryptosporidium: From Molecules to Disease, CABI, Wallingford 2009, pp. 147-157.

[15] Garcia, L. S., Practical Guide to Diagnostic Parasitology, ASM Press, Washington, DC 2009.

[16] Stark, D., Al-Qassab, S. E., Barratt, J. L., Stanley, K., Roberts, T., Marriott, D., Harkness, J., Ellis, J. T., J. Clin. Microbiol. 2011, 49, 257-262.

[17] Cacciò, S. M., Pozio, E., Expert Rev. Anti Infect. Ther. 2006, 4, 429-443.

[18] Stensvold, C. R., Nielsen, H. V., J. Clin. Microbiol. 2012, 50, 540-541.

[19] Johnston, S. P., Ballard, M. M., Beach, M. J., Causer, L., Wilkins, P. P., J. Clin. Microbiol. 2003, 41, 623-626.

[20] Weitzel, T., Dittrich, S., Möhl, I., Adusu, E., Jelinek, T., Clin. Microbiol. Infect. 2006, 12, 656-659.

[21] Jex, A. R., Smith, H. V., Monis, P. T., Campbell, B. E., Gasser, R. B., Biotechnol. Adv. 2008, 26, 304-317.

[22] Ndao, M., Interdiscip. Perspect. Infect. Dis. 2009, 2009, 1-15.

[23] ten Hove, R., van Esbroeck, M., Vervoort, T., van den Ende, J., Van Lieshout, L., Verweij, J., Eur. J. Clin. Microbiol. Infect. Dis. 2009, 28, 1045-1053.

[24] van Lieshout, L., Verweij, J. J., Curr. Opin. Infect. Dis. 2010, 23, 488-493.

[25] Gasser, R. B., Vet. Parasitol. 1999, 84, 229-258.

[26] Gasser, R. B., Vet. Parasitol. 2006, 136, 69-89.

[27] Gasser, R. B., Hu, M., Chilton, N. B., Campbell, B. E., Jex, A. J., Otranto, D., Cafarchia, C., Beveridge, I., Zhu, X., Nature protocols 2006, 1, 3121-3128.

[28] Abs EL-Osta, Y. G., Chalmers, R. M., Gasser, R. B., Mol. Cell. Probes 2003, 17, 127134.

[29] Gasser, R. B., EL-Osta, Y. G. A., Chalmers, R. M., Appl. Environ. Microbiol. 2003, 69, 2719-2730. 
[30] Wu, Z., Nagano, I., Boonmars, T., Nakada, T., Takahashi, Y., Appl. Environ. Microbiol. 2003, 69, 4720-4726.

[31] Gasser, R. B., Abs EL-Osta, Y. G., Prepens, S., Chalmers, R. M., Mol. Cell. Probes 2004, 18, 329-332.

[32] Jex, A. R., Ryan, U. M., Ng, J., Campbell, B. E., Xiao, L., Stevens, M., Gasser, R. B., Electrophoresis 2007, 28, 2818-2825.

[33] Jex, A. R., Whipp, M., Campbell, B. E., Caccio, S. M., Stevens, M., Hogg, G., Gasser, R. B., Electrophoresis 2007, 28, 3875-3883.

[34] Nolan, M. J., Jex, A. R., Mansell, P. D., Browning, G. F., Gasser, R. B., Electrophoresis 2009, 30, 2640-2647.

[35] Nolan, M. J., Jex, A. R., Pangasa, A., Young, N. D., Campbell, A. J., Stevens, M., Gasser, R. B., Electrophoresis 2010, 31, 287-298.

[36] Nolan, M. J., Jex, A. R., Upcroft, J. A., Upcroft, P., Gasser, R. B., Electrophoresis 2011, 32, 2075-2090.

[37] Gasser, R. B., Vet. Parasitol. 2012, (In Press) doi:10.1016/j.vetpar.2012.1012.1031.

[38] Young, K. H., Bullock, S. L., Melvin, D. M., Spruill, C. L., J. Clin. Microbiol. 1979, 10, 852-853.

[39] Sauch, J. F., Appl. Environ. Microbiol. 1985, 50, 1434-1438.

[40] Garcia, L. S., Brewer, T. C., Bruckner, D. A., Shimizu, R. Y., Clin. Microbiol. Newsl. 1983, 5, 60-62.

[41] Pontiroli, A., Travis, E. R., Sweeney, F. P., Porter, D., Gaze, W. H., Mason, S., Hibberd, V., Holden, J., Courtenay, O., Wellington, E. M. H., PLoS ONE 2011, 6, e17916.

[42] Kosch, T., Summers, K., Mol. Ecol. Res. 2013, 13, 230-236.

[43] Strong, W. B., Gut, J., Nelson, R. G., Infect. Immun. 2000, 68, 4117-4134.

[44] Mallon, M., MacLeod, A., Wastling, J., Smith, H., Reilly, B., Tait, A., J. Mol. Evol. 2003, 56, 407-417.

[45] Sulaiman, I. M., Fayer, R., Bern, C., Gilman, R. H., Trout, J. M., Schantz, P. M., Das, P., Lal, A. A., Xiao, L., Emerg. Infect. Dis. 2003, 9, 1444-1452.

[46] Gasser, R. B., Chilton, N. B., Hoste, H., Beveridge, I., Nucleic Acids Res. 1993, 21, 2525-2526.

[47] Liu, Q., Sommer, S., BioTechniques 1995, 18, 470-477.

[48] Larkin, M., Blackshields, G., Brown, N., Chenna, R., McGettigan, P., McWilliam, H., Valentin, F., Wallace, I., Wilm, A., Lopez, R., Bioinformatics 2007, 23, 2947-2948.

[49] Maddison, W., Maddison, D., Mesquite: A Modular System for Evolutionary Analysis, 2011, v2.75, (http://mesquiteproject.org).

[50] Huelsenbeck, J. P., Ronquist, F., Bioinformatics 2001, 17, 754-755.

[51] Darriba, D., Taboada, G. L., Doallo, R., Posada, D., Nat. Methods 2012, 9, 772.

[52] Pangasa, A., Jex, A. R., Campbell, B. E., Bott, N. J., Whipp, M., Hogg, G., Stevens, M. A., Gasser, R. B., Mol. Cell. Probes 2009, 23, 10-15.

[53] Ng, J., MacKenzie, B., Ryan, U., Exp. Parasitol. 2010, 125, 348-356.

[54] Waldron, L. S., Dimeski, B., Beggs, P. J., Ferrari, B. C., Power, M. L., Appl. Environ. Microbiol. 2011, 77, 7757-7765.

[55] Waldron, L. S., Ferrari, B. C., Cheung-Kwok-Sang, C., Beggs, P. J., Stephens, N., Power, M. L., Appl. Environ. Microbiol. 2011, 77, 7766-7771.

[56] Xiao, L., Exp. Parasitol. 2010, 124, 80-89.

[57] Jex, A. R., Gasser, R. B., Biotechnol. Adv. 2010, 28, 17-26. 
[58] Jex, A. R., Chalmers, R. M., Smith, H. V., Widmer, G., McDonald, V., Gasser, R. B., in: Palmer, S. R., Soulsby, L., Torgerson, P., Brown, D. W. G. (Eds.), Oxford Textbook of Zoonoses: Biology, Clinical Practice, and Public Health Control, Oxford University Press, Oxford 2011, pp. 536-568.

[59] Xiao, L., Feng, Y., FEMS Immunol. Med. Microbiol. 2008, 52, 309-323.

[60] Yang, R., Lee, J., Ng, J., Ryan, U., Int. J. Parasitol. 2010, 40, 293-297.

[61] Read, C., Walters, J., Robertson, I., Thompson, R., Int. J. Parasitol. 2002, 32, 229-231.

[62] Feng, Y., Xiao, L., Clin. Microbiol Rev. 2011, 24, 110-140.

[63] Kohli, A., Bushen, O. Y., Pinkerton, R. C., Houpt, E., Newman, R. D., Sears, C. L., Lima, A. A. M., Guerrant, R. L., Trans. R. Soc. Trop. Med. Hyg. 2008, 102, 718-725.

[64] Lebbad, M., Petersson, I., Karlsson, L., Botero-Kleiven, S., Andersson, J. O., Svenungsson, B., Svärd, S. G., PLoS Negl. Trop. Dis. 2011, 5, e1262.

[65] Prociv, P., Luke, R. A., Med. J. Aust. 1995, 162, 150-154.

[66] Holt, D. C., McCarthy, J. S., Carapetis, J. R., Int. J. Parasitol. 2010, 40, 1119-1126.

[67] Sturrock, H. J. W., Picon, D., Sabasio, A., Oguttu, D., Robinson, E., Lado, M., Rumunu, J., Brooker, S., Kolaczinski, J. H., PLoS Negl. Trop. Dis. 2009, 3, e537.

[68] Koroma, J. B., Peterson, J., Gbakima, A. A., Nylander, F. E., Sahr, F., Magalhães, R. J. S., Zhang, Y., Hodges, M. H., PLoS Negl. Trop. Dis. 2010, 4, e891.

[69] Carroll, S., Grove, D., Trans. R. Soc. Trop. Med. Hyg. 1986, 80, 406-411.

[70] Ngui, R., Lim, Y. A. L., Traub, R., Mahmud, R., Mistam, M. S., PLoS Negl. Trop. Dis. 2012, 6, e1522.

[71] Palmer, C. S., Traub, R. J., Robertson, I. D., Hobbs, R. P., Elliot, A., While, L., Rees, R., Thompson, R., Vet. Parasitol. 2007, 145, 304-313.

[72] Ghadirian, E., Arfaa, F., J. Parasitol. 1973, 59, 1144-1145.

[73] Hildreth, M., Harmon, A., in: Liu, D. (Ed.), Molecular Detection of Human Parasitic Pathogens, CRC Press, Boca Raton 2012, pp. 571-582.

[74] Robinson, R. A., in: Macpherson, C. N., Meslin, F. X., Wandeler, A. I. (Eds.), Dogs, Zoonoses, and Public Health, CABI, New York 2001, pp. 273-297.

[75] Abeywardena, H., Jex, A. R., Nolan, M. J., Haydon, S. R., Stevens, M. A., McAnulty, R. W., Gasser, R. B., Infect. Genet. Evol. 2012, 12, 1984-1993.

[76] Nolan, M. J., Jex, A. R., Koehler, A. V., Haydon, S. R., Stevens, M. A., Gasser, R. B., Water Res. 2013, 47, 1726-1740. 
Table 1. Summary of the species and/or subgenotypes of Cryptosporidium identified by PCR-based SSCP analysis and targeted sequencing in the present study.

\begin{tabular}{|c|c|c|c|}
\hline Species & Subgenotype & Number & Percentage \\
\hline \multicolumn{4}{|c|}{ Cryptosporidium hominis } \\
\hline & IbA10G2 & 53 & 74.6 \\
\hline & IdA15G1 & 12 & 16.9 \\
\hline & $\operatorname{IgA} 17$ & 4 & 5.6 \\
\hline & $\operatorname{Ig} \mathrm{A} 18$ & 1 & 1.4 \\
\hline & IfA $13 \mathrm{G} 1$ & 1 & 1.4 \\
\hline \multicolumn{4}{|c|}{ Cryptosporidium parvum } \\
\hline & IIaA18G3R1 & 10 & 47.6 \\
\hline & IIaA17G2R1 & 5 & 23.8 \\
\hline & IIaA15G2R1 & 3 & 14.3 \\
\hline & IIaA19G4R1 & 2 & 9.6 \\
\hline & IIaA20G3R1 & 1 & 4.8 \\
\hline \multicolumn{4}{|c|}{ Cryptosporidium meleagridis } \\
\hline & & 2 & 100 \\
\hline
\end{tabular}

Total: 94 


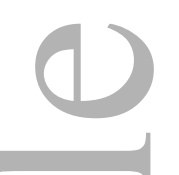

Table 2. Age and gender of the present cohort of 227 people with histories of gastrointestinal disorders, and evidence of infections with Cryptosporidium, Giardia and/or nematodes based on testing using conventional copro-diagnostic techniques

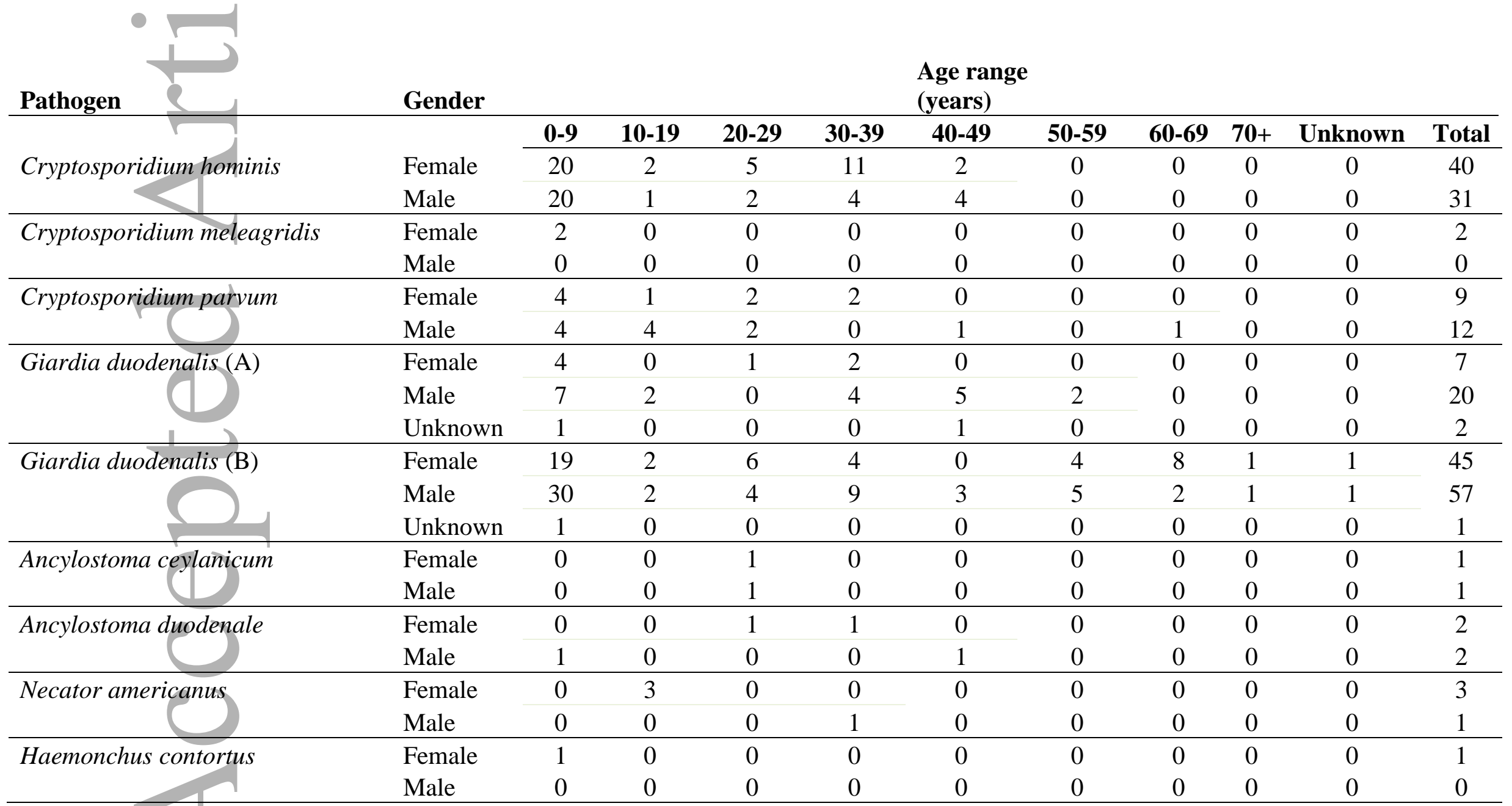


Figure 1. Representative examples of single-strand conformation polymorphism (SSCP) analyses of amplicons. Panel A: ITS-2 amplicons representing Ancylostoma ceylanicum (lanes 1 and 3-5), Necator americanus (lane 2). Panel B: pgp60 amplicons representing Cryptosporidium parvum IbA10G2, C. hominis IIaA18G3R1, C. hominis IIaA20G3R1, C. hominis IIaA15G2R1 (lanes 1-4) and C. hominis IbA10G2 (lanes 5-8). Subgenotypic classification was made following the sequencing of amplicons.

\section{A}

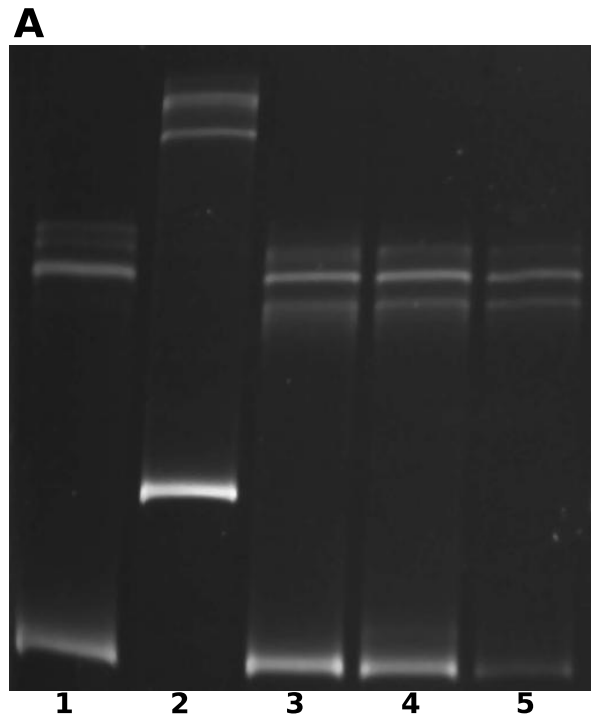

B

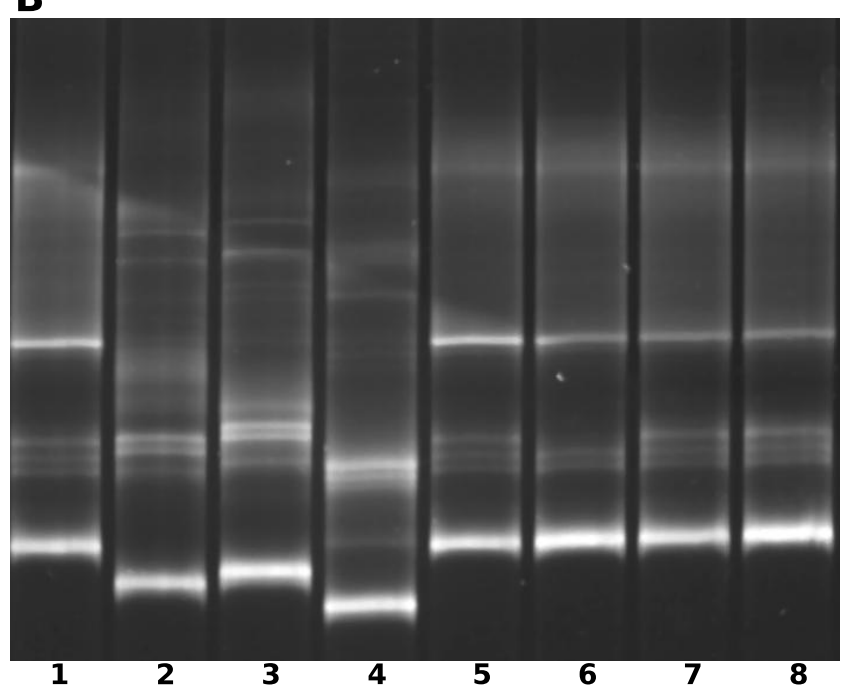


Figure 2. Phylogenetic relationships of Cryptosporidium hominis from humans in the present study (bold-type, followed by sample code) based on analysis of pgp60 sequence data by Bayesian inference; the tree is rooted to $C$. parvum. Posterior probabilities are indicated adjacent to nodes. The number of samples with a particular sequence is in round parentheses.
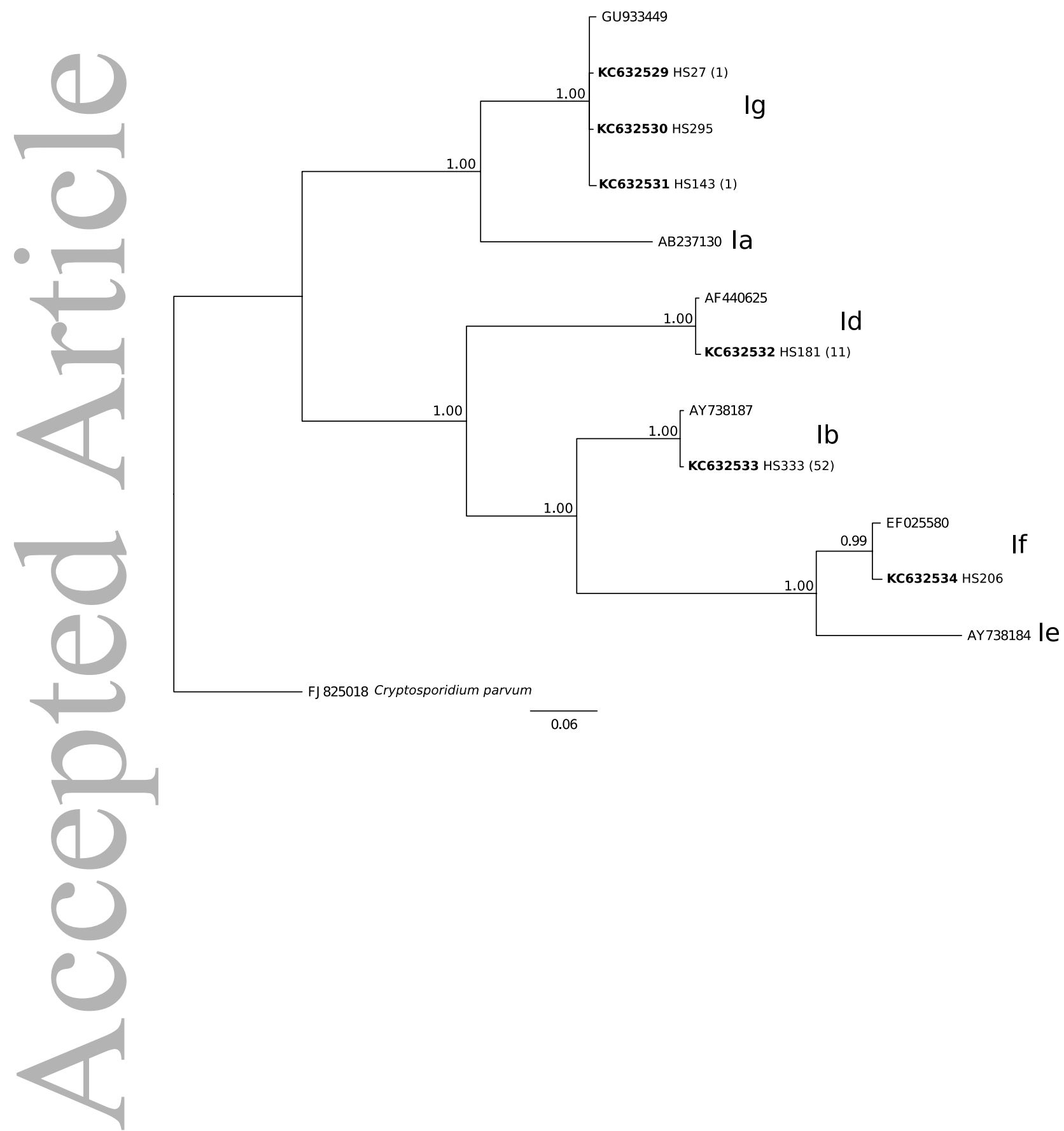
Figure 3. Phylogenetic relationships of Cryptosporidium parvum from humans in the present study (bold-type followed by sample code) based on analysis of pgp60 sequence data by Bayesian inference; the tree is rooted to $C$. hominis. Posterior probabilities are indicated adjacent to nodes. The number of samples with a particular sequence is in round parentheses.

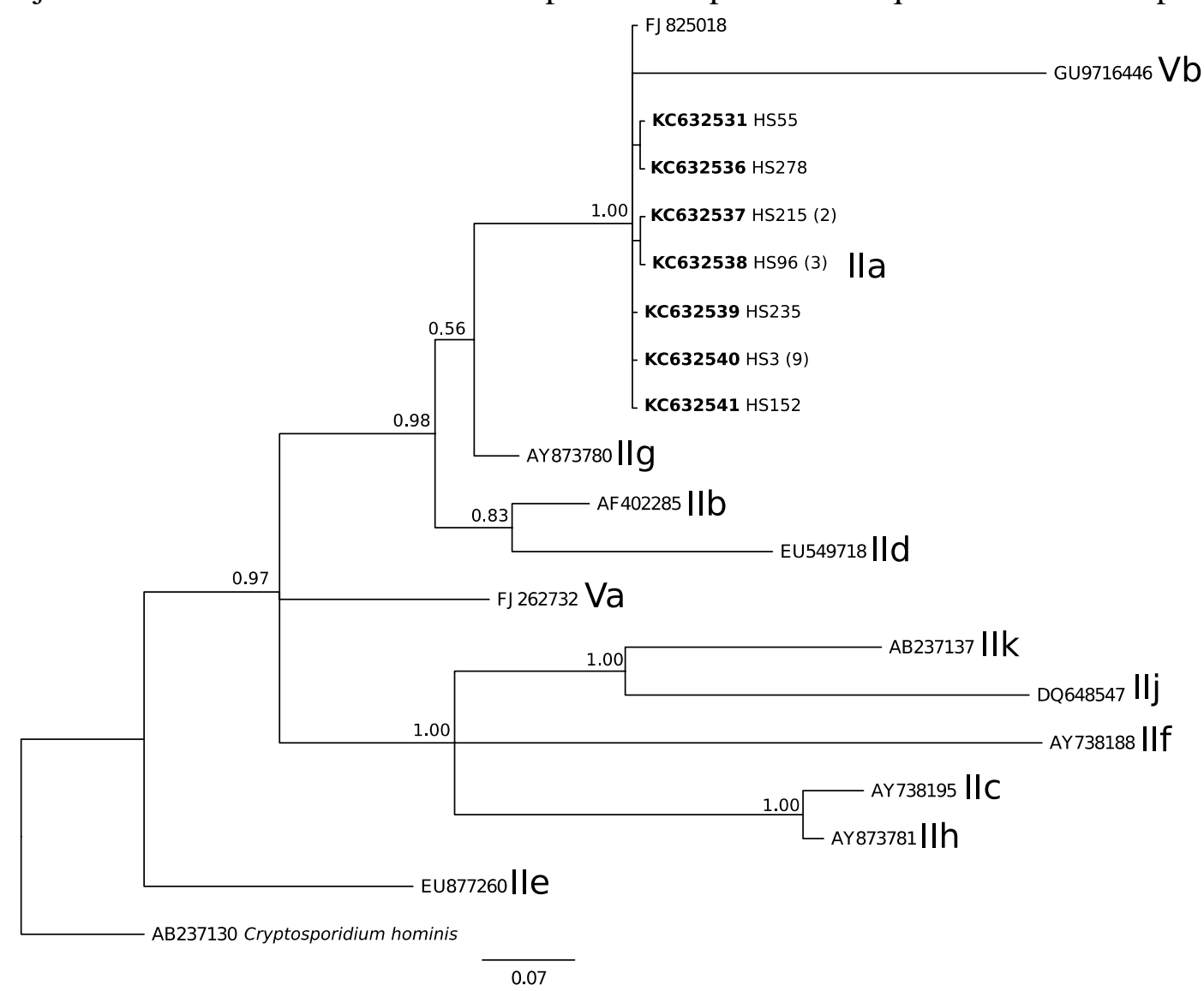


Figure 5. Phylogenetic relationships of strongylid nematodes from humans in the present study (bold-type, followed by sample code) based on analysis of ITS-2 sequence data by Bayesian inference; the tree is rooted to Oesophagostomum dentatum. Posterior probabilities are indicated adjacent to nodes. The number of samples with a particular sequence is in round parentheses.

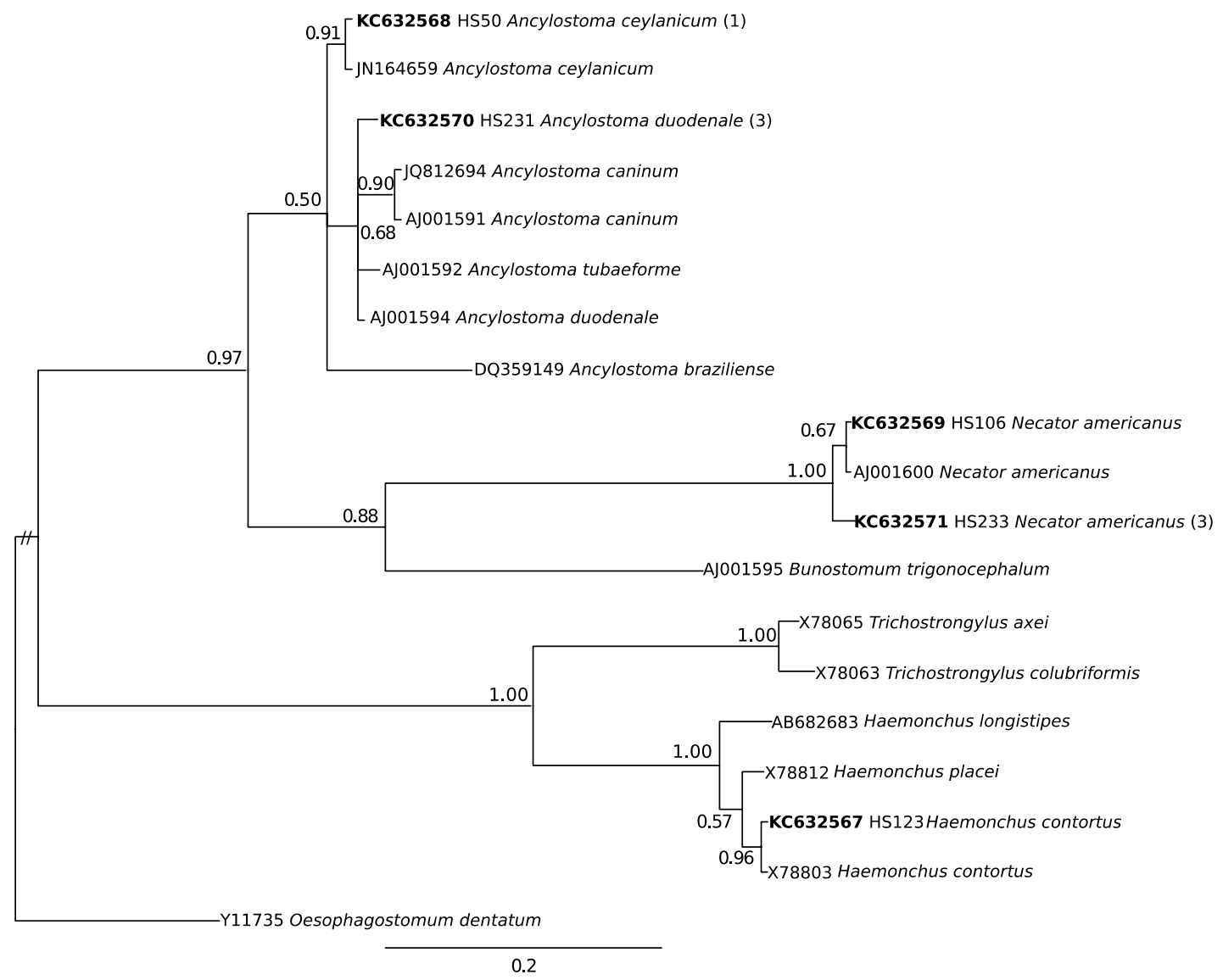

